\title{
ACCELERATING DEEP REINFORCEMENT LEARNING STRATEGIES OF FLOW CONTROL THROUGH A MULTI-ENVIRONMENT APPROACH
}

\author{
Jean Rabault \\ Department of Mathematics \\ University of Oslo \\ jean.rblt@gmail.com
}

\author{
Alexander Kuhnle \\ University of Cambridge \\ alexkuhnle@t-online.de
}

September 17, 2019

\begin{abstract}
Deep Reinforcement Learning (DRL) has recently been proposed as a methodology to discover complex Active Flow Control (AFC) strategies [Rabault, J., Kuchta, M., Jensen, A., Réglade, U., \& Cerardi, N. (2019): "Artificial neural networks trained through deep reinforcement learning discover control strategies for active flow control", Journal of Fluid Mechanics, 865, 281-302]. However, while promising results were obtained on a simple $2 \mathrm{D}$ benchmark flow at a moderate Reynolds number, considerable speedups will be required to investigate more challenging flow configurations. In the case of DRL trained with Computational Fluid Dynamics (CFD) data, it was found that the CFD part, rather than training the Artificial Neural Network, was the limiting factor for speed of execution. Therefore, speedups should be obtained through a combination of two approaches. The first one, which is well documented in the literature, is to parallelize the numerical simulation itself. The second one is to adapt the DRL algorithm for parallelization. Here, a simple strategy is to use several independent simulations running in parallel to collect experiences faster. In the present work, we discuss this solution for parallelization. We illustrate that perfect speedups can be obtained up to the batch size of the DRL agent, and slightly suboptimal scaling still takes place for an even larger number of simulations. This is, therefore, an important step towards enabling the study of more sophisticated Fluid Mechanics problems through DRL.
\end{abstract}

\section{Introduction}

Active Flow Control (AFC) is a problem of considerable theoretical and practical interest, with many applications including drag reduction on vehicles and airplanes (Pastoor et al. 2008, You \& Moin, 2008; Li et al., 2019a b), or optimization of the combustion processes taking place in engines (Wu et al., 2018). Unfortunately, the difficulty of finding efficient strategies for performing AFC is well-known (Brunton \& Noack, 2015; Duriez et al., 2016). It arises from the combination of non-linearity, time-dependence, and high dimensionality inherent to the Navier-Stokes equations.

The importance of AFC is apparent from the large body of literature that discusses both theoretical, computational, and experimental aspects of the problem. In particular, active flow control has been discussed in simulations using both Reduced Order Models and harmonic forcing (Bergmann et al. . 2005), direct simulations coupled with the adjoint method (Flinois \& Colonius, 2015) or linearized models (Lee et al.,2001), and mode tracking methods (Queguineur et al. 2019). Realistic actuation mechanisms, such as plasma actuators (Sato et al. 2015), suction mechanisms (Wang et al. 2016), transverse motion (Li \& Aubry, 2003), periodic oscillations (Lu et al., 2011), oscillating foils (Bao \& Tao, 2013), air jets (Zhu et al., 2019), or Lorentz forces in conductive media (Breuer et al., 2004), are also discussed in details, as well as limitations imposed by real-wold systems (Belson et al., 2013). Similarly, a lot of experimental work has been performed, with the goal of controlling either the cavitation instability (Che et al., 2019), the vortex flow behind a conical forebody (Meng et al. 2018), or the flow separation over a circular cylinder (Jukes \& Choi, 2009abb). 
Finally, while most of the literature has focused on complex, closed-loop control methods, some open-loop methods have also been discussed, both in simulations (Meliga et al., 2010) and in experiments (Shahrabi, 2019).

However, it is still challenging to apply active flow control to situations of industrial interest. By reviewing the literature presented in the previous paragraph one can observe that, while there are good candidates for both sensors and actuators to be used in such systems, finding algorithms for using those measurements and actuation possibilities in an efficient way to perform AFC is challenging. In addition, challenges such as disturbances inherent to the real world, imperfections in the building of the sensors or actuators, and adaptivity to changing external conditions may also be part of the problem. Therefore, the main limitation to AFC is currently the lack of robust, efficient algorithms that can leverage the physical devices available for performing such control. The difficulty of finding efficient algorithms for control and interaction with real world situations or complex systems is also present in other fields of research such as speech recognition (Jelinek, 1997), image analysis (Tarr \& Bülthoff, 1998), or playing complex games such as the game of Go or poker. In those domains, great progress has recently been obtained through the use of data-driven methods and machine learning, and problems that had been unattainable for several decades have been practically solved in recent years (Graves et al. 2013, Krizhevsky et al., 2012; Silver et al., 2017, Brown \& Sandholm, 2019).

Since data-driven and learning-based methods are suitable to be used on non-linear, high dimensional, complex problems, they are, therefore, also promising for performing AFC (Duriez et al. |2016). More specifically, such promising methods include Genetic Programming (GP) (Gautier et al. , 2015;, Duriez et al., 2016), and Deep Reinforcement Learning (DRL) (Verma et al. 2018, Rabault et al..,2018, 2019). Those two methods are among the most successful data-driven approaches at performing nonlinear control tasks in the recent years, and are regarded as possible solutions to the challenges faced by AFC (Duriez et al. 2016). In addition, one of their key properties is their ability to naturally scale, in a parallel fashion, to large amounts of data and / or computational power, which enables very efficient training. This is a well known fact for GP, due to its inherently parallel nature. However, it is maybe slightly less known that the same is true also of DRL, if a suitable implementation is used. In the present work, we illustrate how parallelization of DRL can be performed by extending the work presented in Rabault et al. (2019), and we provide a flexible implementation that will be available open-source. Therefore, we provide both implementations and guidelines that may be used by future work as a basis for further investigation of the application of DRL to AFC in complex scenarios.

In the following, we first provide a short reminder of the Computational Fluid Dynamics (CFD) simulation used, and of the main principles behind DRL. Then, we explain how the data collection part of the algorithm can be parallelized in a general way. Finally, we present the scaling results obtained and we discuss their significance for the use of DRL in more challenging AFC situations

\section{Methodology}

In this section, we summarize the methodology for the CFD simulation (subsection 2.1) and the DRL algorithm (subsection 2.2). Those are identical to what was already used in Rabault et al. (2019). In addition, we present the method used for parallelizing the DRL (subsection 2.3), which is the new contribution of this work.

\subsection{Simulation environment}

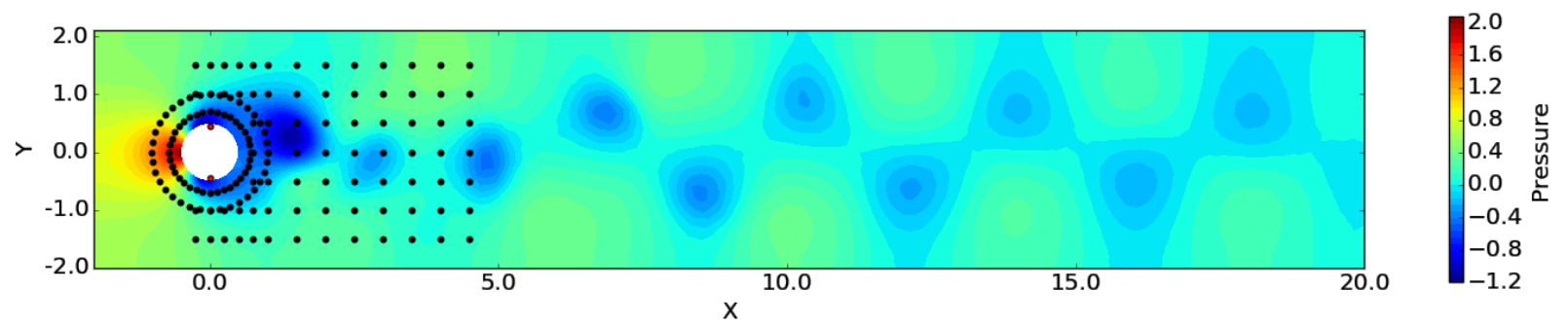

Figure 1: Unsteady non-dimensional pressure wake behind the cylinder after flow initialization without active control. The location of the pressure probes is indicated by the black dots. The location of the control jets is indicated by the red dots. This illustrates the configuration used to perform learning.

The CFD simulation is identical to the one presented in Rabault et al. (2019), and the reader interested in more details should refer to this work and the open-source code implementation: https://github.com/jerabaul29/ 
Cylinder2DFlowControlDRL. To summarize, the CFD case chosen is a simple 2D simulation of the nondimensionalized flow around a cylinder as described by the incompressible Navier-Stokes equations at $R e=100$. The configuration chosen is a copy of a classical benchmark used for the validation of numerical codes (Schäfer et al., 1996). In addition, two small jets of angular width $10^{\circ}$ are set on the sides of the cylinder and inject fluid in the direction normal to the cylinder surface, following the control setup by the Artificial Neural Network (ANN). This implies that the control relies on influencing the separation of the Kármán vortices, rather than direct injection of momentum as could be obtained through propulsion. The jets are controlled through their mass flow rates, respectively $Q_{1}$ and $Q_{2}$. We choose to use synthetic jets, meaning that no net mass flow rate is injected in the flow, which translates to the constraint $Q_{1}+Q_{2}=0$. The general configuration of the simulation is presented in Fig. 1]

The governing Navier-Stokes equations are solved in a segregated manner (Valen-Sendstad et al. . 2012). More precisely, the Incremental Pressure Correction Scheme (IPCS method, Goda (1979)) with an explicit treatment of the non-linear term is used. Spatial discretization then relies on the finite element method implemented within the FEniCS framework (Logg et al., 2012).

The aim of the control strategy is guided by the reward function fed to the DRL during training. In the present work, we want to minimize the drag $D$ through a reduction in the strength of the vortex shedding, in a way analogous to what is obtained by boat tailing (Rabault et al. 2019). This means, in practice, that we are looking for strategies that use the jets as a way to control the vortex shedding process, rather than a way to perform propulsion or converting some of the drag into lift through curbing of the wake. For this, we define the reward function $r$ from both the $\operatorname{drag} D$ and the lift $L$, following:

$$
r=\langle D\rangle_{S}-\left|\langle L\rangle_{S}\right|
$$

where $\langle\bullet\rangle_{S}$ indicates the mean over an action step of the ANN (see section 2.2). In Eq. (1), the term related to drag penalizes large drag values (which are negative due to conventions used in the code, i.e. a larger drag value is more negative than a smaller one), while the term related to lift is here to penalize asymmetric wakes that could be obtained from consistently biased blowing by the jets in one direction. Such consistently biased blowing strategies can be found when no lift penalization is used (see Appendix B), but are not desired as they generate large lift values in addition to the reduced drag. In the figures, we present the value of the drag coefficient, which is a normalized value of the drag $C_{D}=\frac{-D}{\rho \bar{U}^{2} R}$, where $\bar{U}=2 U(0) / 3$ is the mean velocity magnitude, $\rho$ the volumetric mass density of the fluid, and $R$ the diameter of the cylinder.

\subsection{Artificial Neural Network and Deep Reinforcement Learning algorithm}

Artificial intelligence and particularly machine learning have become very attractive fields of research following several recent high-profile successes of Deep Artificial Neural Networks (DANNs), such as attaining super-human performance at image labeling (LeCun et al. 2015), crushing human professionals at the game of Go (Silver et al., 2017), or achieving control of complex robots (Gu et al. 2017), which have shed light on their ability to handle complex, non-linear systems. Those new techniques are now being applied to other disciplines, such as Fluid Mechanics (Kutz, 2017, Brunton et al. 2019), and novel applications of DANNs have recently been proposed for both analyzing laboratory data (Rabault et al. 2017), formulation of reduced order models (Srinivasan et al. 2019), AFC (Rabault et al. 2019), and the control of stochastic systems from only partial observations (Bucci et al. 2019). In particular, the Deep Reinforcement Learning (DRL) approach is a promising avenue for the control of complex systems, including AFC. This approach leverages DANNs to optimize interaction with the system it should control through three channels: observing the state of the system, acting to control the system, and a reward function giving feedback on its current performance. The choice of the reward function allows to direct the efforts of the DANN towards solving a specific problem. In the following, we will use the word "action" to describe the value provided by the ANN at each time step based on a state input, while "control" describes the value effectively used in the simulation.

In the present case, similarly to Rabault et al. (2019), we use a DANN that is a simple fully-connected network, featuring one input layer, two consecutive hidden layers of size 512 each, and one output layer providing the action. The classic rectified linear unit (ReLU) is used as an activation function. The input layer is connected to 151 probes immersed in the simulation that measure the value of the pressure in the vicinity of the cylinder. The output layer provides the action used to obtain the control, i.e. the mass flow rates of the jets. The reward function, presented in Eq. 11, weights both drag and lift to guide the network towards drag-reducing strategies. The DRL algorithm used for training, known in the literature as the Proximal Policy Optimization method (PPO, Schulman et al. (2017)), is among the state-of-the-art for training DANNs to perform continuous control. Each training episode (an episode is a sequence of interations between the DANN and an independent simulation, generating data to be fed to the PPO algorithm) is started from a 
converged, well-defined Kármán vortex street. In the following an episode has a duration corresponding to around 8 vortex shedding periods.

It is difficult for the PPO algorithm to learn the necessity to set time-correlated, continuous actions. This is a consequence of the PPO trying at first purely random actions. Therefore, we added two limitations to the control effectively applied in the CFD simulations. First, the action provided by the network is updated only around typically 10 times per vortex shedding period, i.e. the action is in practise kept constant for a duration of around typically $10 \%$ of the vortex shedding period, except if specifically stated otherwise. This allows the ANN to update its action at a frequency that corresponds roughly to the phenomenon to control, rather than at either a frequency too low (in which case the ANN cannot manage to perform any control), or too high (in which case the randomness from exploration noise does not lead to a sufficient net effect on the system, which can reduce the learning speed). This is a well-known necessity in the literature, and is analogous to the 'frame skip' used in the control of Atari games, for example (Braylan et al., 2015; Neitz et al. 2018). Second, the control effectively set in the simulation is obtained from the latest action and previous control at each numerical time step so that it is made continuous in time to avoid invalid physical phenomena such as infinite acceleration of the fluid contained in the jets. To this end, the control at each time step in the simulation is obtained for each jet as:

$$
c_{s+1}=c_{s}+\alpha\left(a-c_{s}\right),
$$

where $c_{s}$ is the control of the jet considered at the previous numerical time step, $c_{s+1}$ is the new control, $a$ is the action provided by the PPO agent for the current set of time steps, and $\alpha=0.1$ is a numerical parameter. In the present baseline case, there are 50 updates of the control between two consecutive updates of an action, so the value of $\alpha$ allows a quick convergence of the control to the action, relative to the time between action updates. In practice, the exact value of $\alpha$ has little to say for the performance of the control. The existence of those two time scales, and the update of action versus control, are illustrated in Fig. 2 We provide an illustration of the effect of both the frequency of action updates and the value of $\alpha$ in Appendix C.

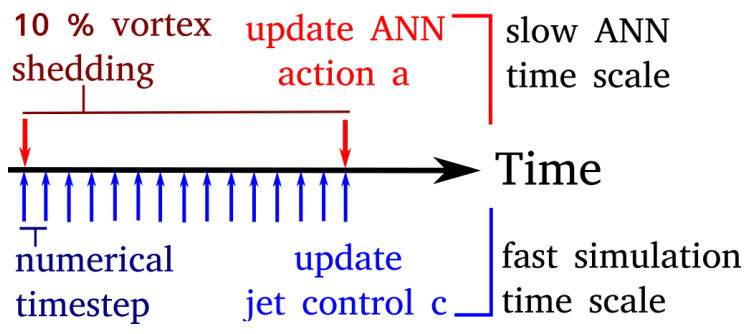

Figure 2: Illustration of the existence of two different time scales in the algorithm. The first one is a fast time scale, which corresponds to the timestep $d t$ of the simulation, and is imposed by considerations around the numerical stability of the simulation. The control applied through the jets (i.e. $c$ ) is updated at this time scale to enforce continuity of the quantities used in the solver. The second time scale is a slower time scale, which corresponds to around $10 \%$ of the vortex shedding period, and captures the relevant time scale of the system. The action set by the ANN is updated following this slower time scale.

\subsection{Parallelization of the data collection for the DRL algorithm}

The code released so far (Rabault et al. (2019), https://github.com/jerabaul29/Cylinder2DFlowControlDRL) allows to train the ANN to perform AFC in about 24 hours using a modern CPU running on a single core. The time needed to perform training is one of the weaknesses of this study, as one would expect that more realistic (and complex) flow simulations may require significantly more learning before an appropriate control strategy is found. Therefore, obtaining speedups is critical for applying this approach to more challenging problems. Benchmarking the code revealed that typically about $99.7 \%$ of the computation time is spent on the environment itself (i.e., the CFD part), rather than the ANN and DRL algorithm. This is due to, on the one hand, the very efficient ANN and DRL implementations provided by TensorFlow / Tensorforce and, on the other hand, the inherent cost associated with CFD. In addition, we note that our hardware uses purely CPUs, and that GPU acceleration may even increase this proportion. Therefore, the optimization effort should focus on the environment part of the code, with Amdahl's Law predicting that full parallelization would allow a theoretical speedup factor of up to typically 300 for this specific case.

There are two different ways of obtaining such speedups: first, one can increase the speed of the CFD simulation itself, i.e. parallelizing the simulation. This topic is well discussed in the literature for a wide range of numerical methods 
(for a short introduction on the topic, one may for example refer to Simon (1992); Gropp \& Smith (1990); Gropp et al. (2001)). However, this approach has its limitations. For example, in our simple 2D simulation, the Finite Element Model (FEM) problem is small enough that our attempts to parallelize the code lead to very limited speedups (no more than a factor of typically less than 2 , independently of using more CPUs), due to the large amount of communication needed between the physical cores compared to the small size of the problems to be solved by the individual cores. This is a well known problem in parallelizing numerical models (Gropp et al., 2001). Therefore, this option is not feasible in our case, and even in the case of more complex CFD simulations it will reach a limit, given enough CPUs are available.

Second, one can attempt to parallelize not the DRL / ANN by themselves (as those use only $0.3 \%$ of the computing time), but the collection of data used to train those algorithms. As the environment itself cannot be sped up much further, another approach is therefore to use several environments, with each being an independent, self-sufficient simulation which feeds data in parallel to the DRL algorithm. This means in practice that the DRL agent learns in parallel from several interactions with simulations. This results in a very simple parallelization technique, which is well-adapted to cases when most of the computational time is spent in the environment itself.

More concretely, the PPO algorithm optimizes the expected return of its stochastic policy, which is in practice estimated based on a number $T_{L}$ of 'rollouts', that is, independent episodes of environment interactions which can be simulated simultaneously. While there exist more sophisticated distributed execution schemes, we observe the following: if the simulation is by far the dominant bottleneck of the training process (which is usually the case in fluid mechanics), the simplest approach is to avoid distributing the (already complex) DRL logic and instead add a lightweight network communication layer on top of the agent-environment interaction. The DRL framework Tensorforce (Kuhnle et al. 2017) supports the capability for DRL models to keep track of parallel streams of experience, which in combination with our environment wrapper allows to parallelize any DRL algorithm for any simulation. In practice, the communication layer is implemented in Tensorforce and associated with a thin wrapper class that communicates through sockets with the different environments. This allows to distribute the environments (i.e., in our case, the expensive CFD simulations), whether on one machine or on a group of machines available through a network if this may be relevant. Those features are all part of the open-source code release (see Appendix A).

We want to emphasize that our main contribution does not consist of a sophisticated parallelization method for DRL training, as indeed even the original PPO paper (Schulman et al., 2017) mentions the parallelized collection of experiences. Instead we observe that recent approaches to distributing DRL (Horgan et al., 2018; Espeholt et al., 2018) are concerned with massive-scale parallelization, based on the HOGWILD! method (Recht et al.,2011). These approaches are useful if the aim is to run 100s of environments simultaneously and if the overhead of message-passing and syncing between different instances, or local cluster of instances, is the bottleneck. Our paper points out that a more straightforward parallelization approach is better suited to the moderately short learning processes but expensive simulations of fluid mechanics problems, which otherwise result in impractical runtimes.

\section{Results and discussion}

The update period of the network is set to $T_{L}=20$ episodes, similarly to what was used in Rabault et al. (2019). This means that the data from 20 episodes are gathered between each learning step, as discussed in the previous section. Therefore, using a number of environments that is a divider of $T_{L}$, together with the synced running mode (meaning that the DRL algorithm waits for all simulations to be finished before starting a new series of simulations), will result in a situation that is effectively identical to the serial learning process. This is illustrated by Fig. 3 by using respectively 1 , 2, 5, 10 and 20 simulation environments. In Fig. 3 three runs are performed in each case. The individual performance curves are indicated by thin lines, while the average of all three runs is indicated by a thicker line. As visible in Fig. 3 (a), the learning curves using 1, 2, 5, 10 and 20 environments running in parallel all collapse on top of each other. This implies that the learning performance is strictly identical between those cases, as we expected. This results in a perfect speedup by a factor equal to the number of environments, as visible in Fig. 3 (a) and (b).

While perfect scaling is obtained in Fig. 3 as expected from the structure of the algorithm, we are interested in exploring the effect of further increasing the number of environments on the training quality. This means, in practice, that we "over-parallelize" the collection of data beyond the DRL algorithm's natural parallelization capabilities. Results are presented in Fig. 4, where we used 32 and 60 environments to investigate the ability of the algorithm to make use of a number of parallel simulations larger than $T_{L}$, corresponding to an over-parallelization of by a factor up to 3 . This case is not equivalent with serial training, and therefore we do not enforce synchronization of episodes. As visible in Fig. 4 . satisfactory speed-up is still observed, although the learning quality is slightly reduced, with the appearance of clear steps in the learning curves.

These steps are due to the fact that several ANN updates take place in a very short time interval, when the environments reach the end of an episode. As this happens more or less at the same time for all environments this means, for example, 


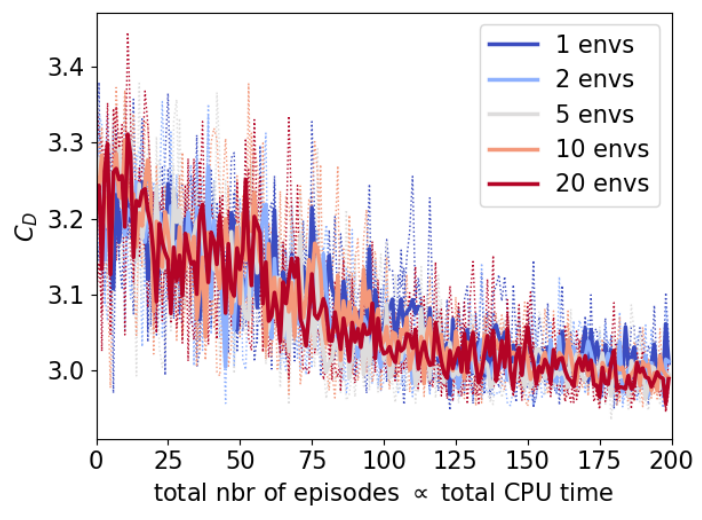

(a)

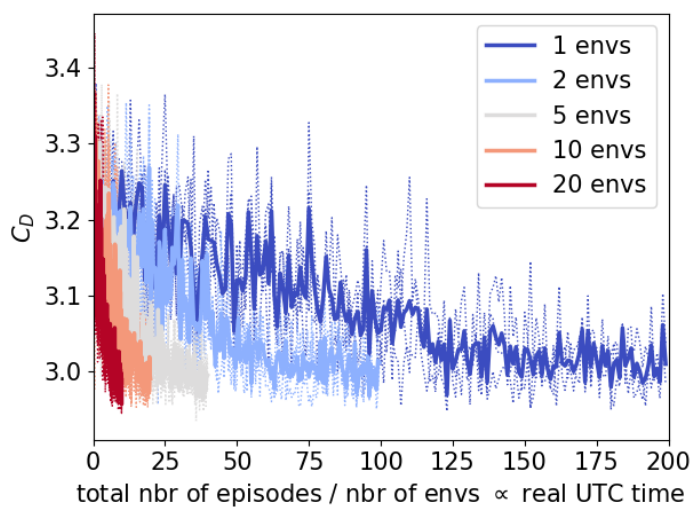

(b)

Figure 3: Scaling results obtained by using a total number of environments that is a divider of the update period $T_{L}$ of the network, in a synced fashion. 3 repetitions are performed for each number of environments. Individual learnings are indicated by thin lines. The average of all 3 learnings in each case is indicated by a thick line. In the present case, the learning is formally identical to the serial case (i.e. with one single environment). This is illustrated by (a), which shows that the shapes of the learning curves are identical independently of the number of environments used. This naturally results in a perfect speedup, as illustrated by both (a) and (b).

that three network updates happen in short succession in case of 60 parallel environments. Overall, this results in a comparatively larger cumulative update of the policy, and that a larger number of episodes pass in between such updates.

It is interesting to note that the second and third of each of those three consecutive updates are actually performed based on data that have been collected following an older policy rather than the current policy at time of the second and third update. Therefore, the second and third updates are based on off-policy data. However, our results indicate empirically that this does not seem to cause problems regarding consistency and stability of the learning algorithm. This can be explained by the fact that deep learning generally follows an iterative approximate as opposed to a fully analytic optimization approach. For instance, policy gradient methods like PPO are based on the assumption that a relatively small number of episode rollouts $T_{L}=20$ approximate the expected reward well. Moreover, PPO specifically already performs multiple small updates within one policy optimization step, thus technically using off-policy data for most of these sub-steps. We conjecture, and observe in our experiments, that such "slightly off-policy" updates do not affect the learning process of on-policy DRL algorithms negatively, which further adds to the effectiveness of our simplistic parallelization approach.

\section{Conclusion}

In this work, we build on the results presented in Rabault et al. (2019) by providing algorithm parallelization improvements and a multi-environment implementation that greatly speeds up the learning. This allows the DRL algorithm to gather simultaneously data from several independent simulations in a parallel fashion. For a number of environments that is a divider of the update period of the ANN, the situation is effectively equivalent to classical serial training, and thus perfect scaling is obtained both theoretically and in practice, up to 20 times in our case. For a number of environments larger than the update period of the ANN, some of the network updates take place off-policy. This, together with timing effects in the policy update, causes steps in the learning curve. However, the PPO algorithm is found to be robust to these "slightly" off-policy updates and learning still takes place almost as expected. We experimentally measure speedups of up to around 60 in this case. We expect there to be a trade-off between extreme over-parallelization and increasingly deteriorating results when comparing to the same effective number of training steps in the serial case.

Those results are an important milestone towards the application of DRL/PPO to Fluid Mechanics problems which are more sophisticated and realistic than the simple benchmark problem of Rabault et al. (2019). Furthermore, the ability to perform training in parallel will allow to perform several valuable parametric studies in a reasonable amount of time, for example exploring the optimal position of pressure sensors or how many inputs are necessary for effective control. The scalings observed here are expected to enable even larger performance increases on more complex problems. 


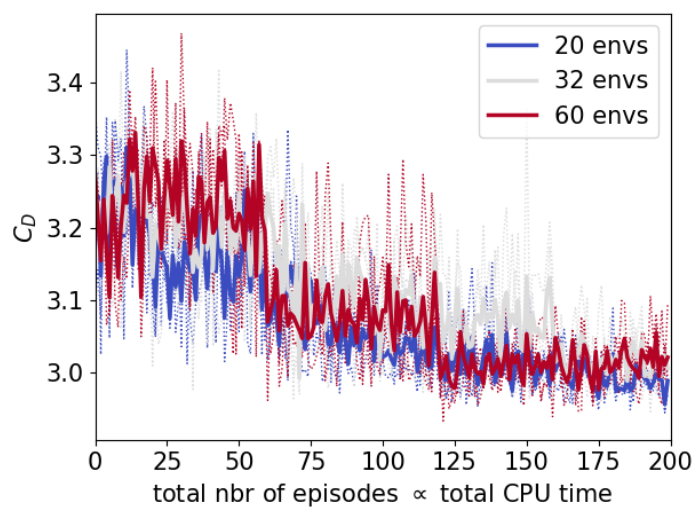

(a)

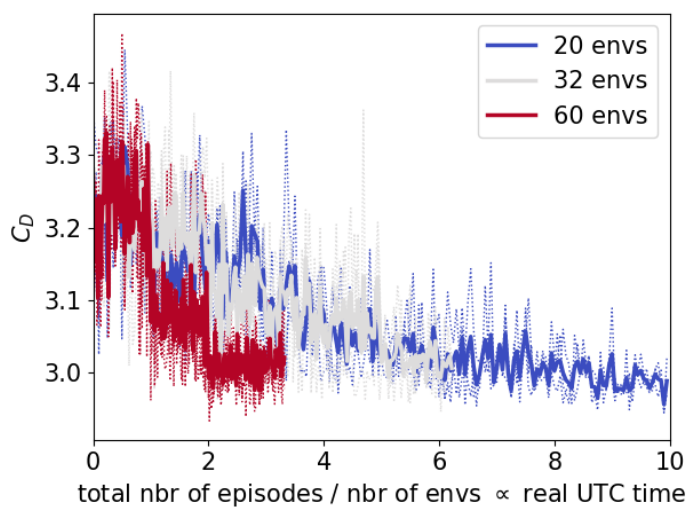

(b)

Figure 4: Scaling results obtained by using a total number of environments that is larger than the update period $T_{L}$. 3 repetitions are performed for each number of environments. Individual learnings are indicated by thin lines. The average of all 3 learnings in each case is indicated by a thick line. As visible in (a), learning still takes place almost satisfactory though steps are clearly visible in the learning curves. This is due to many updates of the network taking place almost simultaneously, when multiple batches of environments reach the end of an episode at about the same time, which consequently triggers a comparatively large cumulative improvement of the policy. While this slightly degrades the learning speed measured in terms of raw number of episodes compared with the equivalent synced case of a smaller number of environments (a), favorable speedups are still observed (b). Note the difference in scale for (b) compared with Fig. 3 (b), and the final speedup factor obtained (around 55 to 60).

Indeed, one usually increases the batch size and the number of episodes between updates together with the underlying complexity of a DRL task, since more trajectories in the phase space are required to generate meaningful gradient updates with higher complexity. In addition, the present parallelization could be combined with the parallelization of the simulation itself, when the size of the underlying problem is suitable. This is also an important point for getting closer to real-world applications.

To summarize, we foresee that the combination of both parallelism methods could allow DRL of AFC through CFD to scale to thousands of CPUs, which opens way to applying the methodology to more challenging, and more realistic, problems. In order to support the development of such methods, all code and implementations are released as open-source (see Appendix A).

\section{Acknowledgement}

The help of Terje Kvernes for setting up the computational infrastructure used in this work is gratefully acknowledged. In addition, we want to thank Dr. Miroslav Kuchta for many interesting discussions and an early version of the socket communication. We gratefully acknowledge help, guidance, and CPU time provided by UH-IaaS (the Norwegian Academic Community Cloud, IT departments, University of Oslo and University of Bergen. http://www.uh-iaas.no/). This work was performed thanks to funding received by the University of Oslo in the context of the 'DOFI' project (grant number 280625).

\section{Appendix A: Open Source code}

The source code of this project, together with a docker container that enforces full reproducibility of our results, is released as open-source on the GitHub of the first author [NOTE: the repository is empty for now, the code will be released upon publication in the peer-reviewed literature]: https://github.com/jerabaul29/Cylinder2DFlowControlDRLParallel. The simulation environment is based on the open-source finite element framework FEniCS (Logg et al. 2012 ) $\mathrm{v}$ 2018.1.0. The PPO agent is based on the open-source implementation provided by Tensorforce (Kuhnle et al. . 2017), which builds on top of the Tensorflow framework (Abadi et al. 2016). This code is an extension of the serial DRL for active flow control available here: https://github.com/jerabaul29/Cylinder2DFlowControlDRL. 


\section{Appendix B: learning with and without lift penalization}

In the case when no lift penalization is used in the equation for the reward Eqn. (1), the DRL algorithm is able to increase its reward through discovering strategies in which a systematic bias is present, i.e. jets blow consistently in the same direction at their maximum strength after a given point in time. This is considered as a 'cheating' strategy, as the drag reduction is accompanied by the production of a large lift. This problem is illustrated in Fig. 5. This problem is effectively suppressed by applying the lift penalization presented in Eqn. (1), since this applies a negative reward to large lift biases introduced by this kind of strategies.

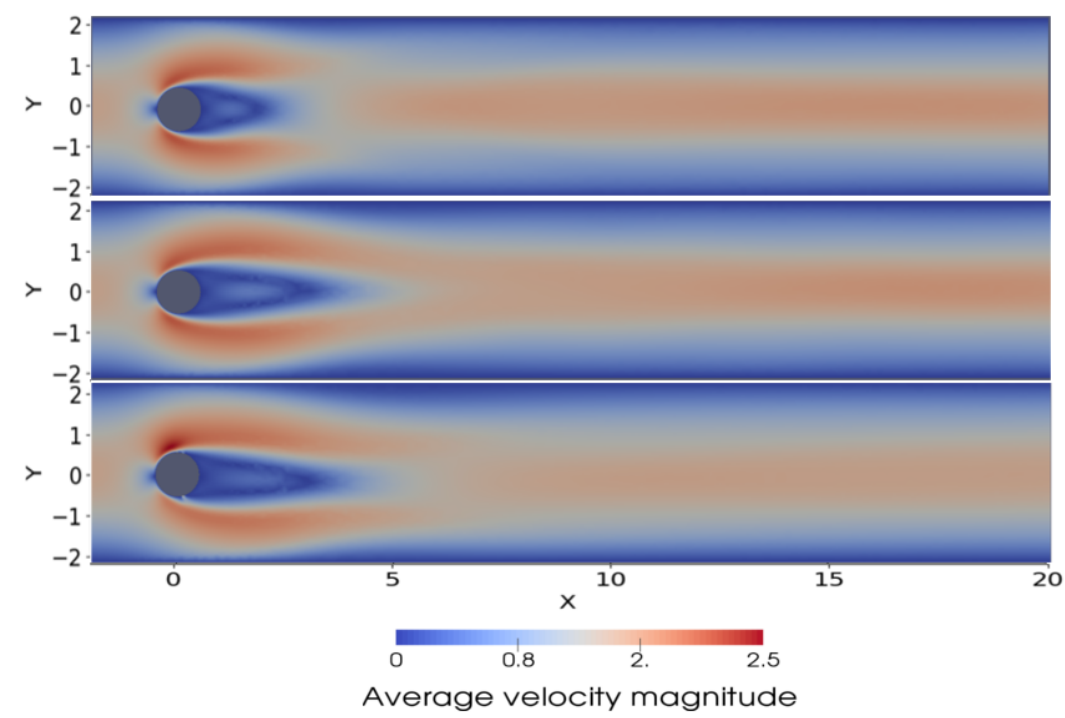

Figure 5: Illustration of the effect of 'cheating' strategies when no lift penalization is present. All velocity maps indicate the velocity magnitude, averaged over around 10 vortex shedding periods. At the top, no control is present. In the middle, control is present, and the DRL algorithm was trained with lift penalization. In this case, control is taking place satisfactorily and the jets are used to control the vortex shedding. At the bottom, control is present and no lift penalization is used during training (in addition, the maximum intensity of the jets is allowed to be larger so that the effect of biased blowing is more visible). In this case, clear asymmetry of the wake and velocity pattern is visible, and the velocity excess present at the top of the cylinder compared to the bottom creates a large mean lift value.

\section{Appendix C: effect of action frequency and control smoothing}

\subsection{Effect of the action update frequency}

As explained in Fig. 2 and section II B, there are two different natural time scales: the first one is very short and corresponds to the numerics of the CFD simulation (typical period associated with the numerics of the simulation: $d t$ ), and the second one is slower and corresponds to the physical dynamics happening in the system (typical period of the Karman vortex shedding: $T_{K}$ ). Therefore, the control applied to the simulation must be applied at an intermediate time scale $T_{a}$ such that $T_{K}>T_{a}>d t$. A bad choice of $T_{a}$ makes learning impossible, as illustrated in Fig. 6 . In the case when $T_{a} / T_{K}=0.5 \%$, the action frequency is too high, and therefore the random exploration noise is applied for a very short time before being updated. This means that the ANN is never able to significantly modify the state of the Karman vortex street and cannot learn. By contrast, in the case when $T_{a} / T_{K}=100 \%$, the action update frequency is too low for the ANN to control anything of the vortex dynamics. In the middle of those values, there is a sweet spot at around $T_{a} / T_{K}=10 \%$ where the frequency of action update is high enough so that the ANN can control the system, but low enough so that the random exploration noise has enough time to act before update so that the system reacts to it in a measurable way.

\subsection{Effect of the smoothing law}

As a consequence of the necessity to choose a relevant action time scale $T_{a}$ that is much larger than the numerical simulation time scale $d t$, one needs to interpolate between action updates to generate the control to use at each time step. 


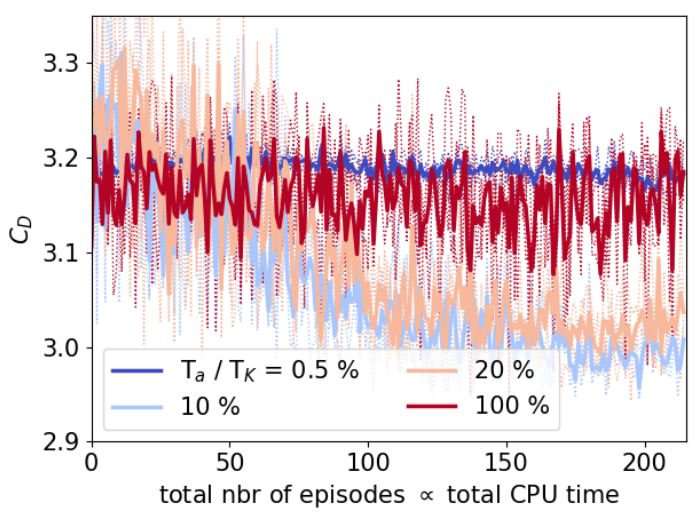

Figure 6: Illustration of the importance of the choice of a relevant time scale for the update of the action set by the network (action update is performed with a period $T_{a}$ ), relatively to the time scale describing the dynamics of the system to control (the Karman alley has a typical time scale described by the period $T_{K}$ ). A too low $T_{a}$ means that the random exploration does not create any consistent forcing that changes the state of the system, and therefore no learning takes place. A too high $T_{a}$ means that the update in the action is too slow to perform a control varying with the phase of the vortex shedding, and therefore no control can take place. In the middle, there is a sweet spot where control is possible and learning takes place. As in the other similar figures, individual learnings are indicated by thin lines. The average of all 3 learnings in each case is indicated by a thick line.

This is schematically shown by Fig. 2. and also explained in section II B. The interpolation can be performed in several fashions, and must follow some conditions of smoothness and continuity to avoid both unphysical phenomena such as infinite accelerations in the fluid, and phenomena of numerical instability. One can for example use an exponential decay law based on the control value from the previous action to the new one, corresponding for example to what is used in Eqn. (2). In this case, which is the one used in this whole paper unless explicitly stated differently, the choice of the decay constant allows to adapt the speed of the convergence of the control towards the value given by the new action. In our case, we find that reasonable values lead to satisfactory learning (see Fig. 7). Other laws can be used, for example linear interpolation between the actions determining the controls. This also works fine, as illustrated in Fig. 7 A careful observation of Fig. 7/ can suggest that the linear interpolation is slightly less efficient than the exponential decay, and this may be a consequence of the fact that the linear interpolation converges to the value of the updated action slower than the exponential decay, which may introduce a form for lag in the control by the ANN. However, this phenomenon is quite minor.

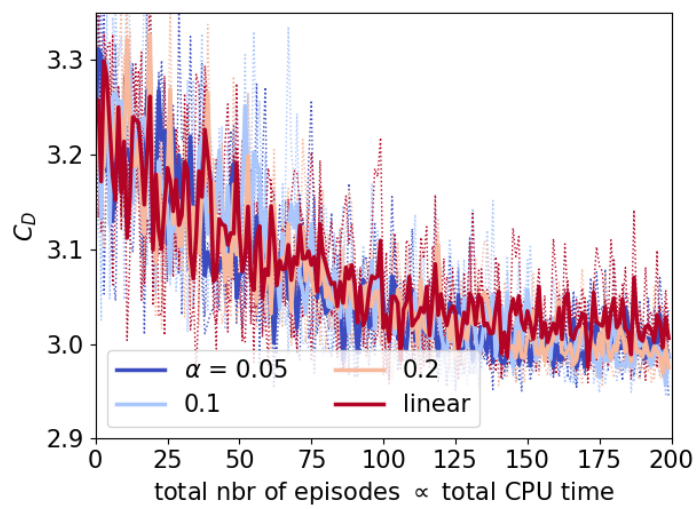

Figure 7: Illustration of the stability of the learning process respectively to the exact value of $\alpha$ and, more generally, the kind of interpolation used for computing the control between action updates by the ANN. As in the other similar figures, individual learnings are indicated by thin lines. The average of all 3 learnings in each case is indicated by a thick line. 


\section{References}

Abadi, Martín, Barham, Paul, Chen, Jianmin, Chen, Zhifeng, Davis, Andy, Dean, JefFrey, Devin, Matthieu, Ghemawat, Sanjay, Irving, Geoffrey, Is ard, Michael \& others 2016 Tensorflow: A system for large-scale machine learning. In OSDI, , vol. 16, pp. 265-283.

BAO, Y. \& TAO, J. 2013 Active control of a cylinder wake flow by using a streamwise oscillating foil. Physics of Fluids 25 (5), 053601, arXiv: https://doi.org/10.1063/1.4802042.

Belson, Brandt A., Semeraro, Onofrio, Rowley, Clarence W. \& Henningson, Dan S. 2013 Feedback control of instabilities in the two-dimensional blasius boundary layer: The role of sensors and actuators. Physics of Fluids 25 (5), 054106, arXiv: https://doi.org/10.1063/1.4804390.

Bergmann, Michel, Cordier, LAurent \& Brancher, JeAn-Pierre 2005 Optimal rotary control of the cylinder wake using proper orthogonal decomposition reduced-order model. Physics of Fluids 17 (9), 097101, arXiv: https://doi.org/10.1063/1.2033624.

Braylan, Alex, Hollenbeck, Mark, Meyerson, Elliot \& Miikkulainen, Risto 2015 Frame skip is a powerful parameter for learning to play atari. In Workshops at the Twenty-Ninth AAAI Conference on Artificial Intelligence.

Breuer, Kenneth S., Park, Jinil \& Henoch, Charles 2004 Actuation and control of a turbulent channel flow using lorentz forces. Physics of Fluids 16 (4), 897-907, arXiv: https://doi.org/10.1063/1.1647142.

Brown, NOAM \& SANDhOlm, TuOMAS 2019 Superhuman ai for multiplayer poker. Science p. eaay2400.

Brunton, Steven, Noack, Bernd \& Koumoutsakos, Petros 2019 Machine learning for fluid mechanics. arXiv preprint arXiv:1905.11075.

Brunton, Steven L \& NoAcK, Bernd R 2015 Closed-loop turbulence control: progress and challenges. Applied Mechanics Reviews 67 (5), 050801.

Bucci, Michele Aless andro, Semeraro, Onofrio, Allauzen, Alexandre, Wisniewski, Guillaume, CORDier, LAURENT \& MATHELIN, LiOnEL 2019 Control of chaotic systems by deep reinforcement learning. arXiv preprint arXiv:1906.07672 .

Che, Bangxiang, Chu, Ning, Cao, Linlin, Schmidt, Steffen J., Likhachev, Dmitriy \& Wu, Dazhuan 2019 Control effect of micro vortex generators on attached cavitation instability. Physics of Fluids 31 (6), 064102 , arXiv: https://doi.org/10.1063/1.5099089.

Duriez, Thomas, Brunton, Steven L \& NoACK, Bernd R 2016 Machine Learning Control-Taming Nonlinear Dynamics and Turbulence. Springer.

Espeholt, Lasse, Soyer, Hubert, Munos, Remi, Simonyan, Karen, Mnih, Vlad, Ward, Tom, Doron, Yotam, Firoiu, Vlad, Harley, Tim, Dunning, Iain, Legg, Shane \& Kavukcuoglu, Koray 2018 IMPALA: Scalable distributed deep-RL with importance weighted actor-learner architectures. In Proceedings of the 35th International Conference on Machine Learning (ed. Jennifer Dy \& Andreas Krause), Proceedings of Machine Learning Research, vol. 80, pp. 1407-1416. Stockholmsmässan, Stockholm Sweden: PMLR.

Flinois, Thibault L. B. \& Colonius, Tim 2015 Optimal control of circular cylinder wakes using long control horizons. Physics of Fluids 27 (8), 087105, arXiv: https://aip.scitation.org/doi/pdf/10.1063/1.4928896.

Gautier, Nicolas, Aider, J-L, Duriez, Thomas, Noack, BR, Segond, Marc \& Abel, Markus 2015 Closed-loop separation control using machine learning. Journal of Fluid Mechanics 770, 442-457.

GodA, Katuнiко 1979 A multistep technique with implicit difference schemes for calculating two- or threedimensional cavity flows. Journal of Computational Physics 30 (1), 76 - 95.

Graves, Alex, Mohamed, AbDel-Rahman \& Hinton, Geoffrey 2013 Speech recognition with deep recurrent neural networks. In 2013 IEEE international conference on acoustics, speech and signal processing, pp. 6645-6649. IEEE.

Gropp, William D, Kaushik, Dinesh K, Keyes, DaVid E \& Smith, Barry F 2001 High-performance parallel implicit cfd. Parallel Computing 27 (4), 337 - 362, parallel computing in aerospace. 
Gropp, William D. \& Smith, EdWARd B. 1990 Computational fluid dynamics on parallel processors. Computers \& Fluids 18 (3), $289-304$.

Gu, S., Holly, E., Lillicrap, T. \& Levine, S. 2017 Deep reinforcement learning for robotic manipulation with asynchronous off-policy updates. In 2017 IEEE International Conference on Robotics and Automation (ICRA), pp. 3389-3396.

Horgan, Dan, Quan, John, Budden, David, BArth-Maron, Gabriel, Hessel, Matteo, van Hasselt, HADO \& SILVER, DAVID 2018 Distributed prioritized experience replay. In 6th International Conference on Learning Representations, ICLR 2018, Vancouver, BC, Canada, April 30 - May 3, 2018, Conference Track Proceedings.

JELINEK, FREDERICK 1997 Statistical methods for speech recognition. MIT press.

Jukes, Timothy N. \& Choi, Kwing-So $2009 a$ Control of unsteady flow separation over a circular cylinder using dielectric-barrier-discharge surface plasma. Physics of Fluids 21 (9), 094106, arXiv: https://doi.org/10.1063/1.3237151.

Jukes, Timothy N. \& Choi, Kwing-So 2009b Flow control around a circular cylinder using pulsed dielectric barrier discharge surface plasma. Physics of Fluids 21 (8), 084103, arXiv: https://doi.org/10.1063/1.3194307.

Krizhevsky, Alex, Sutskever, Ilya \& Hinton, Geoffrey E 2012 Imagenet classification with deep convolutional neural networks. In Advances in neural information processing systems, pp. 1097-1105.

Kuhnle, Alexander, SchaArschmidt, Michael \& Fricke, Kai 2017 Tensorforce: a tensorflow library for applied reinforcement learning. Web page.

KutZ, J. Nathan 2017 Deep learning in fluid dynamics. Journal of Fluid Mechanics 814, 1-4.

LeCun, Yann, Bengio, Yoshua \& Hinton, Geoffrey 2015 Deep learning. Nature 521 (7553), 436.

Lee, Keun H., Cortelezzi, LuCA, Kim, John \& Speyer, Jason 2001 Application of reduced-order controller to turbulent flows for drag reduction. Physics of Fluids 13 (5), 1321-1330, arXiv: https://doi.org/10.1063/1.1359420.

LI, FU \& AUBRY, NADINE 2003 Feedback control of a flow past a cylinder via transverse motion. Physics of Fluids 15 (8), 2163-2176, arXiv: https://doi.org/10.1063/1.1582182.

Li, Ruiying, Borée, Jacques, NoAck, Bernd R., Cordier, Laurent \& Harambat, Fabien $2019 a$ Drag reduction mechanisms of a car model at moderate yaw by bi-frequency forcing. Phys. Rev. Fluids 4, 034604.

Li, Yiqing, Cui, Wenshi, JiA, Qing, Li, Qiliang, YAng, ZhigAng \& NoACK, Bernd R 2019b Optimization of active drag reduction for a slanted ahmed body in a high-dimensional parameter space. arXiv preprint arXiv:1905.12036.

Logg, Anders, Mardal, Kent-Andre \& Wells, GARTH 2012 Automated solution of differential equations by the finite element method: The FEniCS book, , vol. 84. Springer Science \& Business Media.

Lu, Lin, QIn, JiAn-Min, Teng, Bin \& Li, YU-Cheng 2011 Numerical investigations of lift suppression by feedback rotary oscillation of circular cylinder at low reynolds number. Physics of Fluids 23 (3), 033601, arXiv: https://doi.org/10.1063/1.3560379.

Meliga, Philippe, Sipp, Denis \& Chomaz, Jean-Marc 2010 Open-loop control of compressible afterbody flows using adjoint methods. Physics of Fluids 22 (5), 054109, arXiv: https://doi.org/10.1063/1.3425625.

Meng, Xuanshi, Long, Yuexiao, Wang, Jianlei, Liu, Feng \& Luo, Shijun 2018 Dynamics and control of the vortex flow behind a slender conical forebody by a pair of plasma actuators. Physics of Fluids 30 (2), 024101 , arXiv: https://doi.org/10.1063/1.5005514.

Neitz, Alexander, Parascandolo, Giambattista, Bauer, Stefan \& Schölkopf, Bernhard 2018 Adaptive skip intervals: Temporal abstraction for recurrent dynamical models. In Advances in Neural Information Processing Systems, pp. 9816-9826.

Pastoor, Mark, Henning, Lars, NoAck, Bernd R, King, Rudibert \& TAdmor, Gilead 2008 Feedback shear layer control for bluff body drag reduction. Journal of fluid mechanics 608, 161-196. 
Queguineur, Matthieu, Gicquel, L. Y. M., Dupuy, F., Misdaris,, A. \& Staffelbach, G. 2019 Dynamic mode tracking and control with a relaxation method. Physics of Fluids 31 (3), 034101, arXiv: https://doi.org/10.1063/1.5085474.

Rabault, Jean, Kolaas, Jostein \& Jensen, Atle 2017 Performing particle image velocimetry using artificial neural networks: a proof-of-concept. Measurement Science and Technology 28 (12), 125301.

Rabault, Jean, Kuchta, Miroslav, Jensen, Atle, Réglade, Ulysse \& Cerardi, Nicolas 2019 Artificial neural networks trained through deep reinforcement learning discover control strategies for active flow control. Journal of Fluid Mechanics 865, 281-302.

Rabault, Jean, Reglade, Ulysse, Cerardi, Nicolas, Kuchta, MiroslaV \& Jensen, Atle 2018 Deep reinforcement learning achieves flow control of the $2 \mathrm{~d}$ karman vortex street. arXiv preprint arXiv:1808.10754 .

Recht, Benjamin, Re, Christopher, Wright, Stephen \& Niu, Feng 2011 Hogwild: A lock-free approach to parallelizing stochastic gradient descent. In Advances in Neural Information Processing Systems 24 (ed. J. ShaweTaylor, R. S. Zemel, P. L. Bartlett, F. Pereira \& K. Q. Weinberger), pp. 693-701. Curran Associates, Inc.

Sato, Makoto, nonomura, Taku, Okada, Koichi, Asada, Kengo, Aono, Hikaru, Yakeno, Aiko, Abe, Yoshiaki \& FujiI, Kozo 2015 Mechanisms for laminar separated-flow control using dielectricbarrier-discharge plasma actuator at low reynolds number. Physics of Fluids 27 (11), 117101, arXiv: https://aip.scitation.org/doi/pdf/10.1063/1.4935357.

Schäfer, M., Turek, S., Durst, F., Krause, E. \& Rannacher, R. 1996 Benchmark Computations of Laminar Flow Around a Cylinder, pp. 547-566. Wiesbaden: Vieweg+Teubner Verlag.

Schulman, John, Wolski, Filip, Dhariwal, Prafulla, Radford, Alec \& Klimov, Oleg 2017 Proximal policy optimization algorithms. arXiv preprint arXiv:1707.06347 .

Shahrabi, A. F. 2019 The control of flow separation: Study of optimal open loop parameters. Physics of Fluids 31 (3), 035104, arXiv: https://doi.org/10.1063/1.5082945.

Silver, David, Schrittwieser, Julian, Simonyan, Karen, Antonoglou, Ioannis, Huang, Aja, Guez, Arthur, Hubert, Thomas, Baker, Lucas, Lai, Matthew, Bolton, Adrian \& OTHers 2017 Mastering the game of Go without human knowledge. Nature 550 (7676), 354.

SimON, HORST D 1992 Parallel computational fluid dynamics-implementations and results. NASA STI/Recon Technical Report A 94.

Srinivasan, PA, Guastoni, L, Azizpour, Hossein, Schlatter, Philipp \& Vinuesa, Ricardo 2019 Predictions of turbulent shear flows using deep neural networks. Physical Review Fluids 4 (5), 054603.

TARR, Michael J \& BÜlthoff, HeINRICH H 1998 Image-based object recognition in man, monkey and machine. Cognition 67 (1), 1 - 20.

Valen-Sendstad, Kristian, Logg, Anders, Mardal, Kent-Andre, Narayanan, Harish \& Mortensen, MIKAEL 2012 A comparison of finite element schemes for the incompressible navier-stokes equations. In Automated Solution of Differential Equations by the Finite Element Method, pp. 399-420. Springer.

Verma, Siddhartha, Novati, Guido \& Koumoutsakos, Petros 2018 Efficient collective swimming by harnessing vortices through deep reinforcement learning. Proceedings of the National Academy of Sciences, arXiv: http://www.pnas.org/content/early/2018/05/16/1800923115.full.pdf.

Wang, Chenglei, Tang, Hui, Yu, Simon C. M. \& Duan, Fei 2016 Active control of vortex-induced vibrations of a circular cylinder using windward-suction- leeward-blowing actuation. Physics of Fluids 28 (5), 053601, arXiv: https://doi.org/10.1063/1.4947246.

Wu, Zhi, FAn, DeWei, Zhou, Yu, Li, RUIYING \& NoACK, BeRnd R. 2018 Jet mixing optimization using machine learning control. Experiments in Fluids 59 (8), 131.

You, D. \& MoIN, P. 2008 Active control of flow separation over an airfoil using synthetic jets. Journal of Fluids and Structures 24 (8), 1349 - 1357, unsteady Separated Flows and their Control.

Zhu, Honguun, Tang, Tao, Zhao, Honglei \& Gao, Yue 2019 Control of vortex-induced vibration of a circular cylinder using a pair of air jets at low reynolds number. Physics of Fluids 31 (4), 043603, arXiv: https://doi.org/10.1063/1.5092851. 